\title{
Caputo-Katugampola fractional Volterra functional differen- tial equations with a vanishing lag function
}

\author{
M. I. Youssef \\ Department of Mathematics, College of Science, Jouf University, P. O. Box 2014, Sakaka, Saudi Arabia. \\ Department of Mathematics, Faculty of Education, Alexandria University, Alexandria, Egypt.
}

\begin{abstract}
In the present article, we study the solvability of a class of fractional functional integro-differential equations of the CaputoKatugampola type. The existence of solutions is investigated under sufficient conditions as well as the assumptions which guarantee the uniqueness of the solution is explained. Also, we examine the continuous dependence of the solution on the initial condition, the lag function $0 \leqslant \psi(t) \leqslant t$, and the considered nonlinear functional. We give an example to explain our results. The outcomes in this paper extend the results developed by El-Sayed et al. in [A. M. A. El-Sayed, R. G. Ahmed, J. Nonlinear Sci. Appl., 13 (2020), 1-8], recently.
\end{abstract}

Keywords: Volterra functional equation, existence, uniqueness, fixed point principle, delay function.

2010 MSC: 34A12, 45D99, 34K37.

(C)2020 All rights reserved.

\section{Introduction}

Differential and integral equations have become important tools for modeling many real-life phenomena. Also, in ordinary and partial differential equations we can convert several initial and boundary value problems to an equivalent integral equations. In recent years fractional differential and integral models act an essential role in describing various processes in many real-life situations in different fields such as mechanics, mathematical biology, economics, medicine, and many others (see $[2,5,11,12,16,18,21]$ ). As a contribution to the non-fractional approach, El-Sayed et al. studied in [11] the sufficient conditions which guarantee existence, uniqueness, and continuous dependence of solution for a Cauchy problem of a functional differential equation of self-reference $(\psi(t)=t)$, and state-dependence $(\psi(t) \leqslant t)$ on the form

$$
\frac{d y}{d t}=g\left(t, y\left(\int_{0}^{\psi(t)} h(\tau, y(\tau)) d \tau\right)\right), \quad y(0)=y_{0}, \quad t \in[0, T],
$$

in the space of all continuous functions which equipped with the Chebyshev's norm. The authors used the Carathèodory conditions, and Schauder fixed point principle to establish the existence of at least

Email address: miyoussef283@gmail . com (M. I. Youssef)

doi: $10.22436 /$ jnsa.013.05.06

Received: 2019-10-14 Revised: 2020-02-11 Accepted: 2020-02-15 
one continuous solution. The uniqueness of solution was studied as well. Also, they investigated the dependence of the solution on the delay function $\psi(t)$, the functional $h$, and the initial condition $y_{0}$. They gave two examples to demonstrate their results. But the model (1.1) can not tell us what happens to the state $y(t)$ when $t$ is a fractional time. So, in this paper, we shall answer this question. That is we will study the following Caputo-Katugampola fractional nonlinear functional integrodifferential equation

$$
{ }^{C} D_{0^{+}}^{\alpha, \rho} y(t)=g\left(t, y\left(\int_{0}^{\psi(t)} h(\tau, y(\tau)) d \tau\right)\right), \quad 0<t \leqslant T<\infty,
$$

subject to the initial condition

$$
y(0)=y_{0}, \quad y_{0} \in \mathbb{R},
$$

where $0<\alpha<1, \rho$ is a positive real number satisfying $\alpha \rho>1$ and the lag function, $\psi(t)$, is characterized by $\psi(t):=(t-\theta(t))$, with a vanishing lag $\theta(t)$, where $0 \leqslant \theta(t) \leqslant t \forall t \in J:=[0, T]$. The mappings $g(t)$, $\psi(t)$, and $h(t)$ are measurable on J and possess certain attributes which will be decided in Section 3 . It is clear that the equation (1.2) is more general than the equation (1.1), and hence we can derive all the results developed by El-Sayed et al. in [11] as special cases of the present work. The rest of this work is divided as follows. Section 2 presents some definitions, fundamental theorems and auxiliary results we shall need in the subsequent sections. The existence, and uniqueness are studied in Section 3. Section 4 is devoted to the continuous dependence of solution. An illustrative example is given in Section 5 to help clarifying our results. Section 6 is a conclusion.

\section{Preliminaries}

In this section, we shall review some basic definitions and theorems we will need to prove our results. For more details, we refer to $([4,7,17,19,20,22])$.

Definition 2.1 ([14]). Let $g$ be a real valued integrable function defined on $[0, T]$, where $0<T<\infty$. Let $\alpha>0$, and $\rho>0$. Then

$$
\mathrm{I}_{0^{+}}^{\alpha, \rho} g(t):=\frac{\rho^{1-\alpha}}{\Gamma(\alpha)} \int_{0}^{t} \frac{\theta^{\rho-1}}{\left(\mathrm{t}^{\rho}-\theta^{\rho}\right)^{1-\alpha}} g(\theta) d \theta, \quad 0<\mathrm{t} \leqslant \mathrm{T}
$$

is called the left Katugampola fractional integral of order $\alpha$, where $\Gamma(\cdot)$ is the Euler gamma function.

Remark 2.2. The right Katugampola fractional integral can be defined similarly. See $[14,15]$ for the definition. For simplicity, in what follows we will say the Katugampola fractional integral without mentioning the word left. The same remark is applied to the left Katugampola and Caputo-Katugampola fractional derivatives.

Remark 2.3. The Katugampola fractional integral includes the definition of fractional integral due to the Riemann-Liouville and the other one introduced by Hadamard as special cases. Because putting $\rho=$ 1 in Definition 2.1 gives the Riemann-Liouville fractional integral which is utilized in specifying both the Riemann-Liouville and Caputo fractional derivatives. Also, if we assume $\rho \rightarrow 0^{+}$, and applying the L'hospital rule we get the Hadamard fractional integral which is used in describing the Hadamard fractional derivative.

Definition 2.4 ([15]). Let $\mathrm{g}$ be a real valued integrable function defined on $[0, \mathrm{~T}]$, where $0<\mathrm{T}<\infty$. Let $0<\alpha<1$, and $\rho>0$. Then

$$
D_{0^{+}}^{\alpha, \rho} g(t):=t^{1-\rho} \frac{d}{d t}\left(I_{0^{+}}^{1-\alpha, \rho} g(t)\right):=\frac{\rho^{\alpha}}{\Gamma(1-\alpha)} t^{1-\rho} \frac{d}{d t} \int_{0}^{t} \frac{\theta^{\rho-1}}{\left(t^{\rho}-\theta^{\rho}\right)^{\alpha}} g(\theta) d \theta, \quad 0<t \leqslant T
$$

is called the left Katugampola fractional derivative of order $\alpha$. 
Definition 2.5 ([15]). Let $\mathrm{g}$ be a real valued integrable function defined on $[0, \mathrm{~T}]$, where $0<\mathrm{T}<\infty$. Let $0<\alpha<1$, and $\rho>0$. Then

$$
{ }^{C} D_{0^{+}}^{\alpha, \rho} g(t):=D_{0^{+}}^{\alpha, \rho}[g(t)-g(0)], \quad 0<t \leqslant T:=\frac{\rho^{\alpha}}{\Gamma(1-\alpha)} t^{1-\rho} \frac{d}{d t} \int_{0}^{t} \frac{\theta^{\rho-1}}{\left(t^{\rho}-\theta^{\rho}\right)^{\alpha}}[g(\theta)-g(0)] d \theta
$$

is called the left Caputo-Katugampola fractional derivative of order $\alpha$.

Remark 2.6 ([1]). In definition 2.5 if the function $g \in C^{1}([0, T], \mathbb{R})$, then the Caputo-Katugampola fractional derivative of order $\alpha$ takes the form

$$
{ }^{C} D_{0^{+}}^{\alpha, \rho} g(t):=\frac{\rho^{\alpha}}{\Gamma(1-\alpha)} \int_{0}^{t} \frac{1}{\left(t^{\rho}-\theta^{\rho}\right)^{\alpha}} g^{\prime}(\theta) d \theta
$$

Lemma 2.7 ([1]). If the function $\mathrm{g} \in \mathrm{C}([0, \mathrm{~T}], \mathbb{R})$, then ${ }^{\mathrm{C}} \mathrm{D}_{0^{+}}^{\alpha, \rho} \mathrm{I}_{0^{+}}^{\alpha, \rho} \mathrm{g}(\mathrm{t})=\mathrm{g}(\mathrm{t})$.

Lemma 2.8 ([1]). If the function $g \in \mathrm{C}^{1}([0, \mathrm{~T}], \mathbb{R})$, then $\mathrm{I}_{0^{+}}^{\alpha, \rho}{ }^{\mathrm{C}} \mathrm{D}_{0^{+}}^{\alpha, \rho} \mathrm{g}(\mathrm{t})=\mathrm{g}(\mathrm{t})-\mathrm{g}(0)$.

Definition $2.9([3])$. A lag $\theta(t):[0, T] \rightarrow[0, T]$ is said to be a vanishing lag with respect to its domain $[0, T]$ if $\theta(0)=0$, and $\theta(t)>0, \forall t \in(0, T]$.

Definition 2.10 ([13]). Let $\mathrm{H}: \mathrm{Y} \longrightarrow \mathrm{Y}$ be a mapping defined on the Banach space $(\mathrm{Y},\|\cdot\|)$. Then $\mathrm{H}$ is said to be a $p$-contraction if there exists a constant $0 \leqslant p<1$ such that

$$
\left\|H y_{1}-H y_{2}\right\| \leqslant p\left\|y_{1}-y_{2}\right\|, \quad \forall y_{1}, y_{2} \in Y .
$$

Theorem 2.11 ([13]). Let the mapping $\mathrm{H}: \mathrm{Y} \longrightarrow \mathrm{Y}$ be a $\mathrm{p}$-contraction defined on the Banach space $(\mathrm{Y},\|\cdot\|)$. Then the operator $\mathrm{H}$ has a unique fixed point in $\mathrm{Y}$, (i.e. the functional equation $\mathrm{y}=\mathrm{Hy}$ has only one solution in $\mathrm{Y}$ ).

Remark 2.12. Since every closed subspace of a Banach space is also a Banach space, therefore, Theorem 2.11 is still valid if we replace the space $Y$ by a closed subset $\Omega \subseteq Y$, provided that $H(\Omega) \subseteq Y$.

The next theorem is due to the work introduced by Juliusz Schauder and called the Schauder fixed point principle.

Theorem 2.13 ([13]). Let the set $\Omega$ be a convex closed bounded subset of the Banach space $\mathrm{Y}$. Let $\mathrm{H}: \Omega \longrightarrow \mathrm{Y}$ be a continuous operator such that $\mathrm{H} \Omega$ is a compact subset contained in $\Omega$. Then the operator $\mathrm{H}$ has at least one fixed point $\mathrm{y}^{*}$ in $\Omega$.

In what follows let $J:=[0, T]$, and $Y:=C(J, \mathbb{R})$ be the space of all continuous real-valued functions defined on J and endowed with the Chebyshev's norm. That is $\forall y \in Y$ we define $\|y\|=\max _{t \in J}\{|y(t)|\}$. It is clear that $(\mathrm{Y},\|\cdot\|)$ is a Banach space.

\section{The existence and uniqueness results}

Applying the Katugampola fractional integral operator to both sides of equation (1.2), using condition (1.3) and suppose we can use, formally, Lemma 2.8. So, it gives the following nonlinear fractional Volterra integral equation of the CK type.

$$
y(t)=y_{0}+\frac{\rho^{1-\alpha}}{\Gamma(\alpha)} \int_{0}^{t} \frac{\theta^{\rho-1}}{\left(t^{\rho}-\theta^{\rho}\right)^{1-\alpha}} g\left(\theta, y\left(\int_{0}^{\psi(\theta)} h(\tau, y(\tau)) d \tau\right)\right) d \theta
$$

Definition 3.1 ([12]). A Cauchy problem (1.2)-(1.3) is said to have mild solutions if the integral equation (3.1) has solutions and these solutions do not satisfy the initial value problem (1.2)-(1.3). 
We need to assume the following hypotheses:

(V1) The function $g$ is a real valued function defined on $J \times \mathbb{R}$ such that:

(a) the function $\mathrm{g}(\mathrm{t}, \cdot)$ is continuous on $\mathbb{R} \forall \mathrm{t} \in \mathrm{J}$;

(b) there exist two constants $\Lambda_{1}>0$, and $\Lambda_{2}>0$ such that $\forall(t, y) \in J \times \mathbb{R}$ we have $|g(t, y)| \leqslant$ $\Lambda_{1}+\Lambda_{2}|y|$.

(V2) The function $h$ is a positive valued real function defined on $J \times \mathbb{R}$ such that:

(a) the function $h(t, \cdot)$ is continuous on $\mathbb{R} \forall t \in J$;

(b) $|h(t, y)| \leqslant 1 \forall(t, y) \in J \times \mathbb{R}$.

(V3) The delay function is a continuous self-map on J (i.e., the mapping $\psi: J \rightarrow J$ is continuous).

Let us define the constants $M:=\frac{\Lambda_{1}+\Lambda_{2}\left|y_{0}\right|}{T^{1-\alpha \rho} \rho^{\alpha-1} \Gamma(\alpha)-\Lambda_{2} T}$, and $r:=\left|y_{0}\right|+\frac{T M}{\alpha \rho}$. Now we can define the set $\Omega_{r M}:=\{y \in C(J, \mathbb{R}):|y||\leqslant r| y,(t)-y(s)|\leqslant M| t-s \mid, M>0, \forall t, s \in J\}$. It is clear that $\Omega_{r M}$ is a closed bounded convex subset of $C(J, \mathbb{R})$. Let $\mathrm{H}$ be an integral operator defined by

$$
H y(t):=y_{0}+\frac{\rho^{1-\alpha}}{\Gamma(\alpha)} \int_{0}^{t} \frac{\theta^{\rho-1}}{\left(t^{\rho}-\theta^{\rho}\right)^{1-\alpha}} g\left(\theta, y\left(\int_{0}^{\psi(\theta)} h(\tau, y(\tau)) d \tau\right)\right) d \theta
$$

It is easy to prove $H: C(J, \mathbb{R}) \longrightarrow C(J, \mathbb{R})$ under the proposed conditions V1-V3. See ([8-10]) for similar poofs. Now, the solution of the integral equation (3.1) is equivalent to finding a fixed point to the functional equation (3.3)

$$
\mathrm{y}=\mathrm{Hy}
$$

Theorem 3.2. Suppose the hypotheses V1-V3 are fulfilled. Define the integral operator $\mathrm{H}$ from the set $\Omega_{\mathrm{rM}}$ into $\mathrm{C}(\mathrm{J}, \mathbb{R})$. Then the Cauchy problem (1.2)-(1.3) has at least one mild solution $\mathrm{y}^{*} \in \Omega_{\mathrm{rM}}$ provided that $\Lambda_{2} \mathrm{~T}^{\alpha \rho}<$ $\rho^{\alpha-1} \Gamma(\alpha)$.

Proof. First, we will show that $\forall y \in \Omega_{r M}$, and $\forall \mathrm{t} \in \mathrm{J}, \mathrm{Hy}(\mathrm{t}) \in \Omega_{\mathrm{rM}}$. Let $\mathrm{y} \in \Omega_{\mathrm{rM}}, \mathrm{t} \in \mathrm{J}$. So, taking $|\cdot|$ to both sides in equation (3.2), using conditions V1b, V2b, and utilizing the Beta integral implies

$$
\begin{aligned}
|H y(t)| & \leqslant\left|y_{0}\right|+\frac{\rho^{1-\alpha}}{\Gamma(\alpha)} \int_{0}^{t} \frac{\theta^{\rho-1}}{\left(t^{\rho}-\theta^{\rho}\right)^{1-\alpha}}\left|g\left(\theta, y\left(\int_{0}^{\psi(\theta)} h(\tau, y(\tau)) d \tau\right)\right)\right| d \theta \\
& \leqslant\left|y_{0}\right|+\frac{\rho^{1-\alpha}}{\Gamma(\alpha)} \int_{0}^{t} \frac{\theta^{\rho-1}}{\left(t^{\rho}-\theta^{\rho}\right)^{1-\alpha}}\left[\Lambda_{1}+\Lambda_{2}\left|y\left(\int_{0}^{\psi(\theta)} h(\tau, y(\tau)) d \tau\right)-y(0)\right|+\Lambda_{2}|y(0)|\right] d \theta \\
& \leqslant\left|y_{0}\right|+\frac{\rho^{1-\alpha}}{\Gamma(\alpha)} \int_{0}^{t} \frac{\theta^{\rho-1}}{\left(t^{\rho}-\theta^{\rho}\right)^{1-\alpha}}\left[\Lambda_{1}+M \Lambda_{2}\left(\int_{0}^{\psi(\theta)}|h(\tau, y(\tau))| d \tau\right)+\Lambda_{2}|y(0)|\right] d \theta \\
& \leqslant\left|y_{0}\right|+\frac{\rho^{1-\alpha}}{\Gamma(\alpha)} \int_{0}^{t} \frac{\theta^{\rho-1}}{\left(t^{\rho}-\theta^{\rho}\right)^{1-\alpha}}\left[\Lambda_{1}+M \Lambda_{2} \psi(\theta)+\Lambda_{2}\left|y_{0}\right|\right] d \theta \\
& \leqslant\left|y_{0}\right|+\frac{\rho^{1-\alpha}}{\Gamma(\alpha)} \int_{0}^{t} \frac{\theta^{\rho-1}}{\left(t^{\rho}-\theta^{\rho}\right)^{1-\alpha}}\left[\Lambda_{1}+\Lambda_{2}\left(T M+\left|y_{0}\right|\right)\right] d \theta \\
& \leqslant\left|y_{0}\right|+\frac{T^{\alpha \rho} \rho^{-\alpha}\left[\Lambda_{1}+\Lambda_{2}\left(T M+\left|y_{0}\right|\right)\right]}{\Gamma(1+\alpha)} .
\end{aligned}
$$

Substituting $M:=\frac{\Lambda_{1}+\Lambda_{2}\left|y_{0}\right|}{T^{1-\alpha \rho} \rho^{\alpha-1} \Gamma(\alpha)-\Lambda_{2} T}$ and keep in mind that $\Lambda_{2} T^{\alpha \rho}<\rho^{\alpha-1} \Gamma(\alpha) \Longrightarrow M>0$. Then we have $|H y(t)| \leqslant\left|y_{0}\right|+\frac{T M}{\alpha \rho}=r \forall t \in J$, and so $\|y\| \leqslant r$. Now take $t_{1} \in J$, and $t_{2} \in J$ such that $t_{1}<t_{2}$ (without 
loss of generality). Using the assumption $\psi(t) \leqslant t, \forall t \in J$, and applying the Beta integral gives

$$
\begin{aligned}
\left|H y\left(t_{2}\right)-H y\left(t_{1}\right)\right| \leqslant & \frac{\rho^{1-\alpha}}{\Gamma(\alpha)} \int_{0}^{t_{1}}\left(\frac{\theta^{\rho-1}}{\left(t_{2}^{\rho}-\theta^{\rho}\right)^{1-\alpha}}-\frac{\theta^{\rho-1}}{\left(t_{1}^{\rho}-\theta^{\rho}\right)^{1-\alpha}}\right)\left[\Lambda_{1}+M \Lambda_{2} \psi(\theta)+\Lambda_{2}\left|y_{0}\right|\right] d \theta \\
& +\frac{\rho^{1-\alpha}}{\Gamma(\alpha)} \int_{t_{1}}^{t_{2}} \frac{\theta^{\rho-1}}{\left(t_{2}^{\rho}-\theta^{\rho}\right)^{1-\alpha}}\left[\Lambda_{1}+M \Lambda_{2} \psi(\theta)+\Lambda_{2}\left|y_{0}\right|\right] d \theta \\
\leqslant & \frac{\rho^{1-\alpha}\left[\Lambda_{1}+\Lambda_{2}\left(T M+\left|y_{0}\right|\right)\right]}{\Gamma(\alpha)} \int_{0}^{t_{1}}\left(\frac{\theta^{\rho-1}}{\left(t_{2}^{\rho}-\theta^{\rho}\right)^{1-\alpha}}-\frac{\theta^{\rho-1}}{\left(t_{1}^{\rho}-\theta^{\rho}\right)^{1-\alpha}}\right) d \theta \\
& +\frac{\rho^{1-\alpha}\left[\Lambda_{1}+\Lambda_{2}\left(T M+\left|y_{0}\right|\right)\right]}{\Gamma(\alpha)} \int_{t_{1}}^{t_{2}} \frac{\theta^{\rho-1}}{\left(t_{2}^{\rho}-\theta^{\rho}\right)^{1-\alpha}} d \theta \\
\leqslant & \frac{\rho^{-\alpha}\left[\Lambda_{1}+\Lambda_{2}\left(T M+\left|y_{0}\right|\right)\right]}{\Gamma(1+\alpha)}\left[t_{2}^{\alpha \rho}-\left(t_{2}^{\rho}-t_{1}^{\rho}\right)^{\alpha}-t_{1}^{\alpha \rho}+\left(t_{2}^{\rho}-t_{1}^{\rho}\right)^{\alpha}\right] \\
\leqslant & \frac{\rho^{-\alpha}\left[\Lambda_{1}+\Lambda_{2}\left(T M+\left|y_{0}\right|\right)\right]}{\Gamma(1+\alpha)}\left|t_{2}^{\alpha \rho}-t_{1}^{\alpha \rho}\right| .
\end{aligned}
$$

Since $\alpha \rho>1$, so we can apply the inequality $\left|t_{2}^{\alpha \rho}-t_{1}^{\alpha \rho}\right| \leqslant \alpha \rho t_{2}^{\alpha \rho-1}\left|t_{2}-t_{1}\right|, \forall t_{2} \geqslant t_{1}>0$, see [6].

$$
\begin{aligned}
\left|H y\left(t_{2}\right)-H y\left(t_{1}\right)\right| & \leqslant \frac{\rho^{-\alpha}\left[\Lambda_{1}+\Lambda_{2}\left(T M+\left|y_{0}\right|\right)\right]}{\Gamma(1+\alpha)} \alpha \rho t_{2}^{\alpha \rho-1}\left|t_{2}-t_{1}\right| \\
& \leqslant \frac{\rho^{-\alpha}\left[\Lambda_{1}+\Lambda_{2}\left(T M+\left|y_{0}\right|\right)\right]}{\Gamma(1+\alpha)} \alpha \rho T^{\alpha \rho-1}\left|t_{2}-t_{1}\right|=M\left|t_{2}-t_{1}\right| .
\end{aligned}
$$

Therefore, $t_{2} \rightarrow t_{1} \Longrightarrow \mathrm{Hy}\left(t_{2}\right) \rightarrow H y\left(t_{1}\right)$, and hence the operator $\mathrm{H}$ maps the set $\Omega_{\mathrm{r} M}$ into itself (i.e. $\left.H: \Omega_{r M} \rightarrow \Omega_{r M}\right)$. To prove the continuity of the operator $H$, assume $\left(y_{n}\right)_{n=1}^{\infty}$ be a sequence in the set $\Omega_{\mathrm{r} M}$ such that $\mathrm{y}_{\mathrm{n}} \rightarrow \mathrm{y}$ in $\Omega_{\mathrm{r} M}$ as $\mathrm{n} \rightarrow \infty$.

$$
\begin{aligned}
& \left|H y_{n}(t)-H y(t)\right| \\
& \quad \leqslant \frac{\rho^{1-\alpha}}{\Gamma(\alpha)} \int_{0}^{t} \frac{\theta^{\rho-1}}{\left(t^{\rho}-\theta^{\rho}\right)^{1-\alpha}}\left|g\left(\theta, y_{n}\left(\int_{0}^{\psi(\theta)} h\left(\tau, y_{n}(\tau)\right) d \tau\right)\right)-g\left(\theta, y\left(\int_{0}^{\psi(\theta)} h(\tau, y(\tau)) d \tau\right)\right)\right| d \theta .
\end{aligned}
$$

Now from the conditions $V 1 a$, and $V 2 a$, the functions $g$, and $h$ are continuous in $y \forall t \in J$. So, $y_{n} \rightarrow$ $y \Longrightarrow g\left(t, y_{n}\right) \rightarrow g(t, y)$, and $h\left(t, y_{n}\right) \rightarrow h(t, y)$, as $n \rightarrow \infty$. Now

$$
\begin{aligned}
\left|y_{n}\left(\int_{0}^{\psi(\theta)} h\left(\tau, y_{n}(\tau)\right) d \tau\right)-y\left(\int_{0}^{\psi(\theta)} h(\tau, y(\tau)) d \tau\right)\right| & \leqslant\left|y_{n}\left(\int_{0}^{\psi(\theta)} h\left(\tau, y_{n}(\tau)\right) d \tau\right)-y_{n}\left(\int_{0}^{\psi(\theta)} h(\tau, y(\tau)) d \tau\right)\right| \\
& +\left|y_{n}\left(\int_{0}^{\psi(\theta)} h(\tau, y(\tau)) d \tau\right)-y\left(\int_{0}^{\psi(\theta)} h(\tau, y(\tau)) d \tau\right)\right| \\
& \leqslant M \int_{0}^{\psi(\theta)}\left|h\left(\tau, y_{n}(\tau)\right)-h(\tau, y(\tau))\right| d \tau+\frac{\epsilon}{2} \\
& \leqslant M T \frac{\epsilon}{2 M T}+\frac{\epsilon}{2}=\epsilon, \text { as } n \rightarrow \infty .
\end{aligned}
$$

Applying the Lebesgue dominated convergence theorem after using the hypotheses V1 and V2 implies

$$
\begin{aligned}
\lim _{n \rightarrow \infty} H y_{n}(t) & =\lim _{n \rightarrow \infty} \frac{\rho^{1-\alpha}}{\Gamma(\alpha)} \int_{0}^{t} \frac{\theta^{\rho-1}}{\left(t^{\rho}-\theta^{\rho}\right)^{1-\alpha}} g\left(\theta, y_{n}\left(\int_{0}^{\psi(\theta)} h\left(\tau, y_{n}(\tau)\right) d \tau\right)\right) \\
& =\frac{\rho^{1-\alpha}}{\Gamma(\alpha)} \int_{0}^{t} \frac{\theta^{\rho-1}}{\left(t^{\rho}-\theta^{\rho}\right)^{1-\alpha}} g\left(\theta, \lim _{n \rightarrow \infty} y_{n}\left(\int_{0}^{\psi(\theta)} h\left(\tau, y_{n}(\tau)\right) d \tau\right)\right) \\
& =\frac{\rho^{1-\alpha}}{\Gamma(\alpha)} \int_{0}^{t} \frac{\theta^{\rho-1}}{\left(t^{\rho}-\theta^{\rho}\right)^{1-\alpha}} g\left(\theta, y\left(\int_{0}^{\psi(\theta)} h(\tau, y(\tau)) d \tau\right)\right)=H y(t) .
\end{aligned}
$$


So when $n \rightarrow \infty$ the inequality (3.6) becomes less than $\epsilon$ and consequently the operator $\mathrm{H}$ is continuous. Let $\left(H y_{n}\right)_{n=1}^{\infty} \subseteq H \Omega_{r M}$. Let $t_{1} \in J$ and $t_{2} \in J$ with $t_{2}>t_{1}$ such that $\left(t_{2}-t_{1}\right)<\delta$. Applying similar debate like what we have done in the inequality (3.5) implies

$$
\begin{aligned}
\left|H y_{n}\left(t_{2}\right)-H y_{n}\left(t_{1}\right)\right| & \leqslant \frac{\rho^{-\alpha}\left[\Lambda_{1}+\Lambda_{2}\left(T M+\left|y_{0}\right|\right)\right]}{\Gamma(1+\alpha)}\left|t_{2}^{\alpha \rho}-t_{1}^{\alpha \rho}\right| \\
& \leqslant \frac{\rho^{-\alpha}\left[\Lambda_{1}+\Lambda_{2}\left(T M+\left|y_{0}\right|\right)\right]}{\Gamma(1+\alpha)} \alpha \rho T^{\alpha \rho-1}\left|t_{2}-t_{1}\right| \\
& =M\left|t_{2}-t_{1}\right| \rightarrow 0, \text { when } t_{2} \rightarrow t_{1}, \forall n \in \mathbb{N} .
\end{aligned}
$$

Therefore, the sequence $\left(H y_{n}\right)_{n=1}^{\infty}$ is equicontinuous. The sequence $\left(\mathrm{H}_{n}\right)_{n=1}^{\infty}$ is uniformly bounded as well because $\left|\mathrm{H}_{\mathrm{n}}(\mathrm{t})\right| \leqslant \mathrm{r}, \forall \mathrm{n} \in \mathbb{N}$, and $\mathrm{t} \in \mathrm{J}$. From the Arzela Ascoli theorem, we can find a uniformly convergent subsequence $\left(y_{n_{k}}\right)_{n_{k}=1}^{\infty}$ in $\left(y_{n}\right)_{n=1}^{\infty} \subseteq H \Omega_{r M}$ which proves the compactness of the set $H \Omega_{r M}$. Now the operator $\mathrm{H}$ satisfies all conditions of the Schauder fixed point principle. Then the integral operator $H$ has at least on fixed point in the set $\Omega_{r M}$ and consequently the Cauchy problem (1.2)-(1.3) has at least one mild solution in $\Omega_{\mathrm{rM}}$.

Theorem 3.3. Assume the conditions $\mathrm{V} 1 \mathrm{~b}, \mathrm{~V} 2 \mathrm{~b}$, and $\mathrm{V} 3$ are fulfilled. Define the integral operator $\mathrm{H}$ from the set $\Omega_{\mathrm{rM}}$ into $\mathrm{C}(\mathrm{J}, \mathbb{R})$. Suppose there exist two constants $\mathrm{L}_{1}>0, \mathrm{~L}_{2}>0$ such that:

$$
\begin{aligned}
& \left|g\left(t, y_{2}\right)-g\left(t, y_{1}\right)\right| \leqslant L_{1}\left|y_{2}-y_{1}\right|, \quad \forall y_{1}, y_{2} \in \Omega_{r M}, t \in J \\
& \left|h\left(t, y_{2}\right)-h\left(t, y_{1}\right)\right| \leqslant L_{2}\left|y_{2}-y_{1}\right|, \quad \forall y_{1}, y_{2} \in \Omega_{r M}, t \in J .
\end{aligned}
$$

Then the Cauchy problem (1.2)-(1.3) has a unique mild solution $\mathrm{y}^{*} \in \Omega_{\mathrm{rM}}$ provided that

$$
\mathrm{L}_{1} \mathrm{~L}_{2} \mathrm{MT}+\mathrm{L}_{1}<\mathrm{T}^{-\alpha \rho} \rho^{\alpha} \Gamma(1+\alpha) .
$$

Proof. Using the debate we used to deduce equations (3.4) and (3.5) we can prove $H \Omega_{\mathrm{rM}} \subseteq \Omega_{\mathrm{rM}}$. Let $\mathrm{y}_{1}$ and $y_{2}$ be two functions in the set $\Omega_{\mathrm{r} M}$. So we have

$$
\begin{aligned}
& \left|H y_{2}(t)-H y_{1}(t)\right| \\
& \leqslant \frac{\rho^{1-\alpha}}{\Gamma(\alpha)} \int_{0}^{t} \frac{\theta^{\rho-1}}{\left(t^{\rho}-\theta^{\rho}\right)^{1-\alpha}}\left|g\left(\theta, y_{2}\left(\int_{0}^{\psi(\theta)} h\left(\tau, y_{2}(\tau)\right) d \tau\right)\right)-g\left(\theta, y_{1}\left(\int_{0}^{\psi(\theta)} h\left(\tau, y_{1}(\tau)\right) d \tau\right)\right)\right| d \theta \\
& \leqslant \frac{\rho^{1-\alpha}}{\Gamma(\alpha)} \int_{0}^{t} \frac{\theta^{\rho-1}}{\left(t^{\rho}-\theta^{\rho}\right)^{1-\alpha}}\left|g\left(\theta, y_{2}\left(\int_{0}^{\psi(\theta)} h\left(\tau, y_{2}(\tau)\right) d \tau\right)\right)-g\left(\theta, y_{2}\left(\int_{0}^{\psi(\theta)} h\left(\tau, y_{1}(\tau)\right) d \tau\right)\right)\right| d \theta \\
& +\frac{\rho^{1-\alpha}}{\Gamma(\alpha)} \int_{0}^{t} \frac{\theta^{\rho-1}}{\left(t^{\rho}-\theta^{\rho}\right)^{1-\alpha}}\left|g\left(\theta, y_{2}\left(\int_{0}^{\psi(\theta)} h\left(\tau, y_{1}(\tau)\right) d \tau\right)\right)-g\left(\theta, y_{1}\left(\int_{0}^{\psi(\theta)} h\left(\tau, y_{1}(\tau)\right) d \tau\right)\right)\right| d \theta \\
& \leqslant \frac{\mathrm{L}_{1} \rho^{1-\alpha}}{\Gamma(\alpha)} \int_{0}^{\mathrm{t}} \frac{\theta^{\rho-1}}{\left(\mathrm{t}^{\rho}-\theta^{\rho}\right)^{1-\alpha}}\left|\mathrm{y}_{2}\left(\int_{0}^{\psi(\theta)} h\left(\tau, y_{2}(\tau)\right) d \tau\right)-y_{2}\left(\int_{0}^{\psi(\theta)} h\left(\tau, y_{1}(\tau)\right) d \tau\right)\right| d \theta \\
& +\frac{\mathrm{L}_{1} \rho^{1-\alpha}}{\Gamma(\alpha)} \int_{0}^{\mathrm{t}} \frac{\theta^{\rho-1}}{\left(\mathrm{t}^{\rho}-\theta^{\rho}\right)^{1-\alpha}}\left|\mathrm{y}_{2}\left(\int_{0}^{\psi(\theta)} h\left(\tau, y_{1}(\tau)\right) d \tau\right)-y_{1}\left(\int_{0}^{\psi(\theta)} h\left(\tau, y_{1}(\tau)\right) d \tau\right)\right| d \theta \\
& \leqslant \frac{L_{1} M \rho^{1-\alpha}}{\Gamma(\alpha)} \int_{0}^{t} \frac{\theta^{\rho-1}}{\left(t^{\rho}-\theta^{\rho}\right)^{1-\alpha}}\left(\int_{0}^{\psi(\theta)}\left|h\left(\tau, y_{2}(\tau)\right)-h\left(\tau, y_{1}(\tau)\right)\right| d \tau\right) d \theta+\frac{L_{1} \rho^{-\alpha} T^{\alpha \rho}}{\Gamma(1+\alpha)}\left\|y_{2}-y_{1}\right\| \\
& \leqslant \frac{\mathrm{L}_{1} \mathrm{~L}_{2} M \rho^{1-\alpha}}{\Gamma(\alpha)} \int_{0}^{t} \frac{\theta^{\rho-1}}{\left(t^{\rho}-\theta^{\rho}\right)^{1-\alpha}}\left(\int_{0}^{\psi(\theta)}\left|y_{2}(\tau)-y_{1}(\tau)\right| d \tau\right) d \theta+\frac{L_{1} \rho^{-\alpha} T^{\alpha \rho}}{\Gamma(1+\alpha)}\left\|y_{2}-y_{1}\right\| \\
& \leqslant \frac{T^{\alpha \rho} \rho^{-\alpha}\left(L_{1} L_{2} M T+L_{1}\right)}{\Gamma(1+\alpha)}\left\|y_{2}-y_{1}\right\| .
\end{aligned}
$$


Taking the maximum over $t \in J$ and applying the condition $L_{1} L_{2} M T+L_{1}<T^{-\alpha \rho} \rho^{\alpha} \Gamma(1+\alpha)$ implies $\left\|\mathrm{H}_{2}-\mathrm{H} y_{1}\right\| \leqslant p\left\|y_{2}-y_{1}\right\|$, with $p=\frac{T^{\alpha \rho} \rho^{-\alpha}\left(\mathrm{L}_{1} \mathrm{~L}_{2} M T+\mathrm{L}_{1}\right)}{\Gamma(1+\alpha)}<1$. Then the operator $\mathrm{H}$ is $p$-contraction. From Theorem 2.11 with remark 2.12 the operator $\mathrm{H}$ has a unique fixed point in $\Omega_{\mathrm{rM}}$ and thus the Cauchy problem (1.2)-(1.3) has a unique mild solution in $\Omega_{\mathrm{rM}}$.

\section{Dependence of solution on the initial starting point $y(0)$, the lag function $0 \leq \psi(t) \leq t$, and the nonlinear functional $h(t), t \in J$}

In what follows let $y(t):=Y\left(t ; y_{0}, \psi, h\right)$ to indicate that the solution depends on the initial value $y_{0}$, the lag function $\psi$, and the functional $h$. In this part, we shall adapt the definition of continuous dependence stated in [11].

Definition 4.1 ([11]). A solution $y\left(t ; y_{0}, \psi, h\right)$ of the integral equation (3.1) is said to be continuously dependent on the initial value $y_{0}$ if for every $\epsilon>0$, there exists $\delta>0$ depends on $\epsilon$, such that $\left\|y\left(t ; y_{0}, \psi, h\right)-y\left(t ; y_{0}^{*}, \psi, h\right)\right\|<\epsilon, t \in J$, whenever $\left\|y_{0}-y_{0}^{*}\right\|<\delta$.

Lemma 4.2. A solution of an integral equation (3.1) is continuously dependent on the initial starting value $y_{0}$ provided that the conditions of Theorem 3.3 are fulfilled.

Proof. Let $y(t):=y\left(t ; y_{0}, \psi, h\right)$, and $y^{*}(t):=y\left(t ; y_{0}^{*}, \psi, h\right)$ be two solutions of (3.1). So,

$$
\begin{aligned}
y(t)= & y_{0}+\frac{\rho^{1-\alpha}}{\Gamma(\alpha)} \int_{0}^{t} \frac{\theta^{\rho-1}}{\left(t^{\rho}-\theta^{\rho}\right)^{1-\alpha}} g\left(\theta, y\left(\int_{0}^{\psi(\theta)} h(\tau, y(\tau)) d \tau\right)\right) d \theta \\
y^{*}(t)= & y_{0}^{*}+\frac{\rho^{1-\alpha}}{\Gamma(\alpha)} \int_{0}^{t} \frac{\theta^{\rho-1}}{\left(t^{\rho}-\theta^{\rho}\right)^{1-\alpha}} g\left(\theta, y^{*}\left(\int_{0}^{\psi(\theta)} h\left(\tau, y^{*}(\tau)\right) d \tau\right)\right) d \theta \\
\left|y(t)-y^{*}(t)\right| \leqslant & \left|y_{0}-y_{0}^{*}\right| \\
& +\frac{\rho^{1-\alpha}}{\Gamma(\alpha)} \int_{0}^{t} \frac{\theta^{\rho-1}}{\left(t^{\rho}-\theta^{\rho}\right)^{1-\alpha}}\left|g\left(\theta, y\left(\int_{0}^{\psi(\theta)} h(\tau, y(\tau)) d \tau\right)\right)-g\left(\theta, y^{*}\left(\int_{0}^{\psi(\theta)} h\left(\tau, y^{*}(\tau)\right) d \tau\right)\right)\right| d \theta \\
\leqslant & \delta+\frac{L_{1} \rho^{1-\alpha}}{\Gamma(\alpha)} \int_{0}^{t} \frac{\theta^{L_{1} \rho-1}}{\left(t^{\rho}-\theta^{\rho}\right)^{1-\alpha}}\left|y\left(\int_{0}^{\psi(\theta)} h(\tau, y(\tau)) d \tau\right)-y\left(\int_{0}^{\psi(\theta)} h\left(\tau, y^{*}(\tau)\right) d \tau\right)\right| d \theta \\
& +\frac{L_{1} \rho^{1-\alpha}}{\Gamma(\alpha)} \int_{0}^{t} \frac{\theta^{\rho-1}}{\left(t^{\rho}-\theta^{\rho}\right)^{1-\alpha}}\left|y\left(\int_{0}^{\psi(\theta)} h\left(\tau, y^{*}(\tau)\right) d \tau\right)-y^{*}\left(\int_{0}^{\psi(\theta)} h\left(\tau, y^{*}(\tau)\right) d \tau\right)\right| d \theta \\
\leqslant & \left.\delta+\frac{L_{1} L_{2} M \rho^{1-\alpha}}{\Gamma(\alpha)} \int_{0}^{t} \frac{\theta^{\rho-1}}{\left(t^{\rho}-\theta^{\rho}\right)^{1-\alpha}}\left[\int_{0}^{\psi(\theta)}\left|y(\tau)-y^{*}(\tau)\right| d \tau\right)\right] d \theta+\frac{L_{1} \rho^{-\alpha} T^{\alpha \rho}}{\Gamma(1+\alpha)}\left\|y-y^{*}\right\| \\
\leqslant & \delta+\frac{T^{\alpha \rho} \rho^{-\alpha}\left(L_{1} L_{2} M T+L_{1}\right)}{\Gamma(1+\alpha)}\left\|y-y^{*}\right\|=\delta+p\left\|y-y^{*}\right\| .
\end{aligned}
$$

Taking the maximum over $t \in J$, and choosing $\delta=(1-p) \epsilon$, where $p$ is the contraction constant, implies $\left\|y-y^{*}\right\|<\epsilon$, whenever $\left\|y_{0}-y_{0}^{*}\right\|<\delta$. Then the required result follows.

Definition 4.3 ([11]). A solution $Y\left(t ; y_{0}, \psi, h\right)$ of the integral equation (3.1) is said to be continuously dependent on the lag function $\psi$ if for every $\epsilon>0$, there exists $\delta>0$ depending on $\epsilon$, such that $\left\|\mathrm{Y}\left(\mathrm{t} ; \mathrm{y}_{0}, \psi, \mathrm{h}\right)-\mathrm{Y}\left(\mathrm{t} ; \mathrm{y}_{0}, \psi^{*}, \mathrm{~h}\right)\right\|<\epsilon, \mathrm{t} \in \mathrm{J}$, whenever $\left\|\psi-\psi^{*}\right\|<\delta$.

Lemma 4.4. The solution of the integral equation (3.1) is continuously dependent on the lag function $\psi(t), t \in J$, provided that the conditions of Theorem 3.3 are fulfilled. 
Proof. Let $y(t):=y\left(t ; y_{0}, \psi, h\right)$, and $y^{*}(t):=y\left(t ; y_{0}, \psi^{*}, h\right)$ be two solutions of (3.1). So,

$$
\begin{aligned}
& y(t)=y_{0}+\frac{\rho^{1-\alpha}}{\Gamma(\alpha)} \int_{0}^{t} \frac{\theta^{\rho-1}}{\left(t^{\rho}-\theta^{\rho}\right)^{1-\alpha}} g\left(\theta, y\left(\int_{0}^{\psi(\theta)} h(\tau, y(\tau)) d \tau\right)\right) d \theta, \\
& y^{*}(t)=y_{0}+\frac{\rho^{1-\alpha}}{\Gamma(\alpha)} \int_{0}^{t} \frac{\theta^{\rho-1}}{\left(t^{\rho}-\theta^{\rho}\right)^{1-\alpha}} g\left(\theta, y^{*}\left(\int_{0}^{\psi^{*}(\theta)} h\left(\tau, y^{*}(\tau)\right) d \tau\right)\right) d \theta, \\
& \left|y(t)-y^{*}(t)\right| \leqslant \frac{\rho^{1-\alpha}}{\Gamma(\alpha)} \int_{0}^{t} \frac{\theta^{\rho-1}}{\left(t^{\rho}-\theta^{\rho}\right)^{1-\alpha}}\left|g\left(\theta, y\left(\int_{0}^{\psi(\theta)} h(\tau, y(\tau)) d \tau\right)\right)-g\left(\theta, y^{*}\left(\int_{0}^{\psi^{*}(\theta)} h\left(\tau, y^{*}(\tau)\right) d \tau\right)\right)\right| d \theta \\
& \leqslant \frac{L_{1} \rho^{1-\alpha}}{\Gamma(\alpha)} \int_{0}^{t} \frac{\theta^{\rho-1}}{\left(t^{\rho}-\theta^{\rho}\right)^{1-\alpha}}\left|y\left(\int_{0}^{\psi(\theta)} h(\tau, y(\tau)) d \tau\right)-y\left(\int_{0}^{\psi^{*}(\theta)} h\left(\tau, y^{*}(\tau)\right) d \tau\right)\right| d \theta \\
& +\frac{L_{1} \rho^{1-\alpha}}{\Gamma(\alpha)} \int_{0}^{t} \frac{\theta^{\rho-1}}{\left(t^{\rho}-\theta^{\rho}\right)^{1-\alpha}}\left|y\left(\int_{0}^{\psi^{*}(\theta)} h\left(\tau, y^{*}(\tau)\right) d \tau\right)-y^{*}\left(\int_{0}^{\psi^{*}(\theta)} h\left(\tau, y^{*}(\tau)\right) d \tau\right)\right| d \theta \\
& \leqslant \frac{\mathrm{L}_{1} \rho^{-\alpha} T^{\alpha \rho}}{\Gamma(1+\alpha)}\left\|y-y^{*}\right\| \\
& \left.+\frac{\mathrm{L}_{1} M \rho^{1-\alpha}}{\Gamma(\alpha)} \int_{0}^{t} \frac{\theta^{\rho-1}}{\left(t^{\rho}-\theta^{\rho}\right)^{1-\alpha}} \mid \int_{0}^{\psi(\theta)} h(\tau, y(\tau)) d \tau-\int_{0}^{\psi^{*}(\theta)} h\left(\tau, y^{*}(\tau)\right) d \tau\right) \mid d \theta \\
& \leqslant \frac{\mathrm{L}_{1} \rho^{-\alpha} T^{\alpha \rho}}{\Gamma(1+\alpha)}\left\|y-y^{*}\right\| \\
& \left.+\frac{L_{1} M \rho^{1-\alpha}}{\Gamma(\alpha)} \int_{0}^{t} \frac{\theta^{\rho-1}}{\left(t^{\rho}-\theta^{\rho}\right)^{1-\alpha}} \mid \int_{0}^{\psi(\theta)} h(\tau, y(\tau)) d \tau-\int_{0}^{\psi^{*}(\theta)} h(\tau, y(\tau)) d \tau\right) \mid d \theta \\
& \left.+\frac{\mathrm{L}_{1} M \rho^{1-\alpha}}{\Gamma(\alpha)} \int_{0}^{t} \frac{\theta^{\rho-1}}{\left(\mathrm{t}^{\rho}-\theta^{\rho}\right)^{1-\alpha}} \mid \int_{0}^{\psi^{*}(\theta)} h(\tau, y(\tau)) d \tau-\int_{0}^{\psi^{*}(\theta)} h\left(\tau, y^{*}(\tau)\right) d \tau\right) \mid d \theta \\
& \leqslant \frac{\mathrm{L}_{1} \rho^{-\alpha} T^{\alpha \rho}}{\Gamma(1+\alpha)}\left\|y-y^{*}\right\|+\frac{\mathrm{L}_{1} M \rho^{1-\alpha}}{\Gamma(\alpha)} \int_{0}^{\mathrm{t}} \frac{\theta^{\rho-1}}{\left(\mathrm{t}^{\rho}-\theta^{\rho}\right)^{1-\alpha}} \int_{\psi^{*}(\theta)}^{\psi(\theta)}|\mathrm{h}(\tau, \mathrm{y}(\tau))| \mathrm{d} \tau \mathrm{d} \theta \\
& +\frac{\mathrm{L}_{1} \mathrm{~L}_{2} M \rho^{1-\alpha}}{\Gamma(\alpha)} \int_{0}^{\mathrm{t}} \frac{\theta^{\rho-1}}{\left(\mathrm{t}^{\rho}-\theta^{\rho}\right)^{1-\alpha}} \int_{0}^{\psi^{*}(\theta)}\left|\mathrm{y}(\tau)-\mathrm{y}^{*}(\tau)\right| \mathrm{d} \tau \mathrm{d} \theta \\
& \leqslant \frac{\mathrm{L}_{1} M \rho^{-\alpha} T^{\alpha \rho}}{\Gamma(1+\alpha)}\left\|\psi-\psi^{*}\right\|+p\left\|y-y^{*}\right\| \\
& \leqslant \frac{\mathrm{L}_{1} M \rho^{-\alpha} T^{\alpha \rho}}{\Gamma(1+\alpha)} \delta+p\left\|y-y^{*}\right\| .
\end{aligned}
$$

Taking the maximum over $\mathrm{t} \in \mathrm{J}$, and choosing $\delta=\frac{\Gamma(1+\alpha)(1-p)}{\mathrm{L}_{1} M \rho^{-\alpha} T^{\alpha \rho}} \epsilon$ implies $\left\|y-y^{*}\right\|<\epsilon$, whenever $\| \psi-$ $\psi^{*} \|<\delta$. Then the required result follows.

Definition 4.5 ([11]). A solution $Y\left(t ; y_{0}, \psi, h\right)$ of the integral equation (3.1) is said to be continuously dependent on the functional $h$ if for every $\epsilon>0$, there exists $\delta>0$ depending on $\epsilon$, such that $\| Y\left(t ; y_{0}, \psi, h\right)-$ $\mathrm{Y}\left(\mathrm{t} ; \mathrm{y}_{0}, \psi, \mathrm{h}^{*}\right) \|<\epsilon, \mathrm{t} \in \mathrm{J}$, whenever $\left\|\mathrm{h}-\mathrm{h}^{*}\right\|<\delta$.

Lemma 4.6. The solution of the integral equation (3.1) is continuously dependent on the functional $\mathrm{h}(\mathrm{t}), \mathrm{t} \in \mathrm{J}$, provided that the conditions of Theorem 3.3 are fulfilled.

Proof. Let $y(t):=y\left(t ; y_{0}, \psi, h\right)$, and $y^{*}(t):=y\left(t ; y_{0}, \psi, h^{*}\right)$ be two solutions of (3.1). So,

$$
y(t)=y_{0}+\frac{\rho^{1-\alpha}}{\Gamma(\alpha)} \int_{0}^{t} \frac{\theta^{\rho-1}}{\left(t^{\rho}-\theta^{\rho}\right)^{1-\alpha}} g\left(\theta, y\left(\int_{0}^{\psi(\theta)} h(\tau, y(\tau)) d \tau\right)\right) d \theta
$$




$$
\begin{aligned}
y^{*}(t)= & y_{0}+\frac{\rho^{1-\alpha}}{\Gamma(\alpha)} \int_{0}^{t} \frac{\theta^{\rho-1}}{\left(t^{\rho}-\theta^{\rho}\right)^{1-\alpha}} g\left(\theta, y^{*}\left(\int_{0}^{\psi(\theta)} h^{*}\left(\tau, y^{*}(\tau)\right) d \tau\right)\right) d \theta \\
\left|y(t)-y^{*}(t)\right| \leqslant & \frac{\rho^{1-\alpha}}{\Gamma(\alpha)} \int_{0}^{t} \frac{\theta^{\rho-1}}{\left(t^{\rho}-\theta^{\rho}\right)^{1-\alpha}}\left|g\left(\theta, y\left(\int_{0}^{\psi(\theta)} h(\tau, y(\tau)) d \tau\right)\right)-g\left(\theta, y^{*}\left(\int_{0}^{\psi(\theta)} h^{*}\left(\tau, y^{*}(\tau)\right) d \tau\right)\right)\right| d \theta \\
\leqslant & \frac{L_{1} \rho^{1-\alpha}}{\Gamma(\alpha)} \int_{0}^{t} \frac{\theta^{L_{1} \rho-1}}{\left(t^{\rho}-\theta^{\rho}\right)^{1-\alpha}}\left|y\left(\int_{0}^{\psi(\theta)} h(\tau, y(\tau)) d \tau\right)-y\left(\int_{0}^{\psi(\theta)} h^{*}\left(\tau, y^{*}(\tau)\right) d \tau\right)\right| d \theta \\
& +\frac{L_{1} \rho^{1-\alpha}}{\Gamma(\alpha)} \int_{0}^{t} \frac{\theta^{\rho-1}}{\left(t^{\rho}-\theta^{\rho}\right)^{1-\alpha}}\left|y\left(\int_{0}^{\psi(\theta)} h^{*}\left(\tau, y^{*}(\tau)\right) d \tau\right)-y^{*}\left(\int_{0}^{\psi(\theta)} h^{*}\left(\tau, y^{*}(\tau)\right) d \tau\right)\right| d \theta \\
\leqslant & \left.\frac{L_{1} \rho^{-\alpha} T^{\alpha \rho}}{\Gamma(1+\alpha)} \| y-y^{*} \mid+\frac{L_{1} M \rho^{1-\alpha}}{\Gamma(\alpha)} \int_{0}^{t} \frac{\theta^{\rho-1}}{\left(t^{\rho}-\theta^{\rho}\right)^{1-\alpha}}\left[\int_{0}^{\psi(\theta)}\left|h(\tau, y(\tau))-h^{*}\left(\tau, y^{*}(\tau)\right)\right| d \tau\right)\right] d \theta \\
\leqslant & \left.\frac{L_{1} \rho^{-\alpha} T^{\alpha \rho}}{\Gamma(1+\alpha)}\left\|y-y^{*}\right\|+\frac{L_{1} M \rho^{1-\alpha}}{\Gamma(\alpha)} \int_{0}^{t} \frac{\theta^{\rho-1}}{\left(t^{\rho}-\theta^{\rho}\right)^{1-\alpha}}\left[\int_{0}^{\psi(\theta)}\left|h(\tau, y(\tau))-h\left(\tau, y^{*}(\tau)\right)\right| d \tau\right)\right] d \theta \\
& \left.+\frac{L_{1} M \rho^{1-\alpha}}{\Gamma(\alpha)} \int_{0}^{t} \frac{\theta^{\rho-1}}{\left(t^{\rho}-\theta^{\rho}\right)^{1-\alpha}}\left[\int_{0}^{\psi(\theta)}\left|h\left(\tau, y^{*}(\tau)\right)-h^{*}\left(\tau, y^{*}(\tau)\right)\right| d \tau\right)\right] d \theta \\
\leqslant & \frac{L_{1} M \rho^{-\alpha} T^{\alpha \rho+1}}{\Gamma(1+\alpha)}\left\|h-h^{*}\right\|+p\left\|y-y^{*}\right\| \\
\leqslant & \frac{L_{1} M \rho^{-\alpha} T^{\alpha \rho+1}}{\Gamma(1+\alpha)} \delta+p\left\|y-y^{*}\right\| .
\end{aligned}
$$

Taking the maximum over $\mathrm{t} \in \mathrm{J}$, and choosing $\delta=\frac{\Gamma(1+\alpha)(1-p)}{\mathrm{L}_{1} M \rho^{-\alpha} T^{\alpha \rho+1}} \epsilon$, where $\mathrm{p}$ is the contraction constant, implies $\left\|y-y^{*}\right\|<\epsilon$, whenever $\left\|h-h^{*}\right\|<\delta$. Then the required result follows.

\section{Illustrative example}

Example 5.1. Given the following fractional functional initial value problem

$$
{ }^{C} D_{0^{+}}^{\frac{1}{2}, \frac{5}{2}} y(t)=\frac{1}{6}(2 t+3)+\frac{1}{2} y\left(\int_{0}^{q t e^{-t}} \frac{s+\sin (y(s))}{(s+1)^{2}+3 e^{|y(s)|}} d s\right), \quad 0<q<1, t \in(0,1],
$$

subject to the initial staring condition

$$
y(0)=0
$$

Comparing equations 5.1, and 5.2 with equations (1.2), and (1.3) implies $\alpha=\frac{1}{2}, \rho=\frac{5}{2}, \mathrm{~T}=1$, and $\mathrm{y}_{0}=0$. The function $\psi(\mathrm{t})=\mathrm{qt} \mathrm{e}^{-\mathrm{t}}$ is continuous self-map on $[0,1]$ and $\mathrm{qte} \mathrm{e}^{-\mathrm{t}}=\mathrm{t}-\left(1-\mathrm{qe} \mathrm{e}^{-\mathrm{t}}\right) \mathrm{t} \Longrightarrow$ the lag $\theta(t)=\left(1-q e^{-t}\right) t \geqslant 0, \forall t \in[0,1] \Longrightarrow$ the lag is vanishing with respect to $[0,1]$. Furthermore $\psi(0)=0$, and $\psi(t)>0, \forall t \in(0,1] \Longrightarrow \psi(t)$ is a vanishing lag function $\Longrightarrow$ condition V3 is verified. The function $h(t, y)=\frac{t+\sin (y(t))}{(t+1)^{2}+3 e^{|y(t)|}}$ is measurable on $[0,1] \forall y \in \mathbb{R}$, continuous in $y \forall t \in[0,1]$, and moreover $|h(t, y)| \leqslant \frac{1}{2} \Longrightarrow$ condition $V 2$ is satisfied. The function $g(t, y)=\frac{1}{6}(2 t+3)+\frac{1}{2} y\left(\int_{0}^{q t e^{-t}} \frac{s+\sin (y(s))}{(s+1)^{2}+3 e^{|y(s)|}} d s\right)$ is measurable on $[0,1] \forall y \in \mathbb{R}$, continuous in $y \forall t \in$ $[0,1]$, and $|g(t, y)| \leqslant \frac{5}{6}+\frac{1}{2}|y| \Longrightarrow \Lambda_{1}=\frac{5}{6}$, and $\Lambda_{2}=\frac{1}{2} \Longrightarrow$ condition $V 1$ is fulfilled. Now $\rho^{\alpha-1} \Gamma(\alpha)-\Lambda_{2} T^{\alpha \rho}=(2.5)^{-0.5} \Gamma(0.5)-0.5=0.6210>0 \Longrightarrow$ all conditions of Theorem 3.2 are met. Consequently the Cauchy problem (5.1) and (5.2) has at least one continuous mild solution in $\Omega_{\mathrm{rM}}$ with $M=\frac{\Lambda_{1}+\Lambda_{2}\left|y_{0}\right|}{T^{1-\alpha \rho} \rho^{\alpha-1} \Gamma(\alpha)-\Lambda_{2} \mathrm{~T}}=1.3419$, and $r=\left|y_{0}\right|+\frac{\mathrm{TM}}{\alpha \rho}=1.0735$. If the functions $\mathrm{g}$, and $\mathrm{h}$ are Lipschitz in their second independent variable we can guarantee the uniqueness. In this example, we can prove that 
$g(t, y)$ satisfies lipschitz condition with respect to $y$ with constant $L_{1}=\frac{1}{2}$ and $h(t, y)$ is Lipschitz function in $y$ with constant $L_{2}=\frac{1}{4}$ as well. Now $p=\frac{T^{\alpha \rho} \rho^{-\alpha}\left(L_{1} L_{2} M T+L_{1}\right)}{\Gamma(1+\alpha)}=\frac{2.5^{-0.5}[(0.125)(1.3419)+0.5]}{\Gamma(1.5)}=0.4765<1 \Longrightarrow$ all assumptions of Theorem 3.3 are fulfilled. Therefore the initial value problem (1.2)-(1.3) has a unique mild solution in $\Omega_{\mathrm{r} M}$ with $M=1.3419$, and $\mathrm{r}=1.0735$.

\section{Conclusion}

In the current paper, we have investigated a fractional model of the Caputo-Katugampola type. We utilized the Schauder fixed point principle together with the Carathèodory conditions to establish the existence of at least one continuous mild solution under some sufficient weak conditions. We proved the uniqueness of the solution using the Banach fixed point. The results developed by El-Sayed et al. in [11] are special cases of our work. Our work studied the fractional evolution of the model developed by El-Sayed et al. in [11] which was not studied in their work [11].

\section{References}

[1] R. Almeida, A. B. Malinowska, T. Odzijewicz, Fractional Differential Equations With Dependence on the CaputoKatugampola Derivative, J. Comput. Nonlinear Dynam., 11 (2016), 11 pages. 2.6, 2.7, 2.8

[2] T. M. Atanacković, S. Pilipović, B. Stanković, D. Zorica, Fractional Calculus with Applications in Mechanics: Vibrations and Diffusion Processes, John Wiley \& Sons, Hoboken, (2014). 1

[3] A. Bellen, M. Zennaro, Numerical methods for delay differential equations. Numerical Mathematics and Scientific Computation, The Clarendon Press, Oxford University Press, New York, (2003). 2.9

[4] A. Buica, Existence and continuous dependence of solutions of some functional-differential equations, Seminar on Fixed Point Theory (Babes-Bolyai Univ., Cluj-Napoca), 3 (1995), 1-14. 2

[5] V. Daftardar-Gejji, Fractional Calculus and Fractional Differential Equations, Birkhäuser, Singapore, (2019). 1

[6] J. Deng, J. Wang, Existence and approximation of solutions of fractional order iterative differential equations, Central Eur. J. Phys., 11 (2013), 1377-1386. 3

[7] E. Eder, The functional differential equation $x^{\prime}(t)=x(x(t))$, J. Differential Equations, 54 (1984), 390-400. 2

[8] M. M. Elborai, M. A. Abdou, M. I. Youssef, On the existence, uniqueness, and stability behavior of a random solution to a non local perturbed stochastic fractional integro-differential equation, Life Sci. J., 10 (2013), 3368-3376. 3

[9] M. M. Elborai, M. A. Abdou, M. I. Youssef, On some nonlocal perturbed random fractional integro-differential equations, Life Sci. J., 10 (2013), 1601-1609.

[10] M. M. Elborai, M. I. Youssef, On stochastic solutions of nonlocal random functional integral equations, Arab J. Math. Sci., 25 (2019), 180-188. 3

[11] A. M. A. El-Sayed, R. G. Ahmed, Solvability of the functional integro-differential equation with self-reference and statedependence, J. Nonlinear Sci. Appl., 13 (2020), 1-8. 1, 1, 4, 4.1, 4.3, 4.5, 6

[12] M. A. E. Herzallah, Notes on some fractional calculus operators and their properties, J. Fract. Calc. Appl., 5 (2014), 10 pages. $1,3.1$

[13] H. Hochstadt, Integral Equations, John Wiley \& Sons, New York, (1988). 2.10, 2.11, 2.13

[14] U. N. Katugampola, New approach to a generalized fractional integral, Appl. Math. Comput., 218 (2011), 860-865. 2.1, 2.2

[15] U. N. Katugampola, A new approach to generalized fractional derivatives, Bull. Math. Anal. Appl., 6 (2014), 1-15. 2.2, $2.4,2.5$

[16] A. A. Kilbas, H. M. Srivastava, J. J. Trujillo, Theory and applications of fractional differential equations, Elsevier Science B. V., Amsterdam, (2006). 1

[17] E. Kreyszig, Introductory Functional Analysis with Applications, John Wiley \& Sons, New York-London-Sydney, (1978). 2

[18] D. Poljak, C. M. Cvetković, C. V. Dorić, C. I. Zulim, C. Z. Dogaš, C. J. Haueisen, K. E. Drissi, Integral Equation Formulations and Related Numerical Solution Methods in Some Biomedical Applications of Electromagnetic Fields: Transcranial Magnetic Stimulation (TMS), Nerve Fiber Stimulation, Int. J. E-Health Medical Commun., 9 (2018), 65-84. 1

[19] A. Ricardo, Variational Problems Involving a Caputo-Type Fractional Derivative, J. Optim. Theory Appl., 174 (2017), 276-294. 2

[20] S. Stanĕk, Globel properties of solutions of the functional differenatial equation $x(t) x^{\prime}(t)=k x(x(t)), 0<|k|<1$, Funct. Differ. Equ., 9 (2004), 527-550. 2

[21] P. Umamaheswari, K. Balachandran, N. Annapoorani, Existence of solutions of stochastic fractional integrodifferential equations, Discontin. Nonlinearity Complex., 7 (2018), 55-65. 1

[22] S. D. Zeng, D. Baleanu, Y. R. Bai, G. C. Wu, Fractional differential equations of Caputo Katugampola type and numerical solutions, Appl. Math. Comput., 315 (2017), 549-554. 2 